\title{
XMM-Newton and VLT observations of the afterglow of GRB $040827^{\star}$
}

\author{
A. De Luca ${ }^{1}$, A. Melandri ${ }^{2}$, P. A. Caraveo ${ }^{1}$, D. Götz ${ }^{1}$, S. Mereghetti $^{1}$, A. Tiengo ${ }^{1}$, L. A. Antonelli ${ }^{2}$, S. Campana ${ }^{3}$, \\ G. Chincarini ${ }^{3}$, S. Covino ${ }^{3}$, P. D'Avanzo ${ }^{3,4}$, A. Fernandez-Soto ${ }^{5}$, D. Fugazza ${ }^{3}$, D. Malesani ${ }^{6}$, \\ L. Stella ${ }^{2}$, and G. Tagliaferri ${ }^{3}$ \\ 1 INAF - Istituto di Astrofisica spaziale e Fisica Cosmica, sez. di Milano "G. Occhialini”, via Bassini 15, 20133 Milano, Italy \\ e-mail: deluca@mi.iasf.cnr.it \\ 2 INAF - Osservatorio Astronomico di Roma, via di Frascati 33, 00040 Monteporzio Catone (Rome), Italy \\ 3 INAF - Osservatorio Astronomico di Brera, via Bianchi 46, 23807 Merate (LC), Italy \\ ${ }^{4}$ Università degli Studi dell'Insubria, Dipartimento di Fisica e Matematica, via Valleggio 11, 22100 Como, Italy \\ 5 Observatori Astrònomic, Universitat de València, 46100 València, Spain \\ ${ }^{6}$ International School for Advanced Studies (SISSA-ISAS), via Beirut 2-4, 34014 Trieste, Italy
}

Received 15 February 2005 / Accepted 8 May 2005

\begin{abstract}
The field of the Gamma-Ray Burst GRB 040827 was observed with XMM-Newton and with the ESO/VLT starting $\sim 6$ and $\sim 12 \mathrm{~h}$ after the burst, respectively. A fading X-ray afterglow is clearly detected with the XMM-Newton/EPIC instrument, with a time decay $t^{-\delta}$, with $\delta=1.41 \pm 0.10$. Its spectrum is well described by a power law (photon index $\Gamma=2.3 \pm 0.1$ ) affected by an absorption largely exceeding (by a factor $\sim 5$ ) the expected Galactic one, requiring the contribution of an intrinsic, redshifted absorber. In the optical/NIR range, the afterglow emission was observed in the $K_{\mathrm{s}}$ band as a weak source superimposed on the host galaxy, with magnitude $K_{\mathrm{s}}=19.44 \pm 0.13$ ( $12 \mathrm{~h}$ after the GRB, contribution from the host subtracted); in other bands the flux is dominated by the host galaxy. Coupling constraints derived from X-ray spectral fitting and from photometry of the host, we estimated a gas column density in the range $(0.4-2.6) \times 10^{22} \mathrm{~cm}^{-2}$ in the GRB host galaxy, likely located at a redshift $0.5<z<1.7$. GRB 040827 stands out as the best example of an X-ray afterglow with intrinsic absorption.
\end{abstract}

Key words. gamma rays: bursts $-\mathrm{X}$-rays: general

\section{Introduction}

The discovery of afterglow emission at X-ray, optical and radio wavelengths opened a new era in the study of the mysterious Gamma-Ray Burst (GRB) phenomenon. Afterglows' observations yielded most of our current understanding of long $(>2 s)$ GRBs, from their energetics to the evidence of their association with the death of massive stars (see, e.g. van Paradijs et al. 2000; Hurley et al. 2003; Piran 2004, for comprehensive reviews). A fast and accurate localization of GRBs is required for successful follow-up studies of their afterglows. After the end of the BeppoSAX mission, the INTEGRAL satellite proved to be very powerful for such task, thanks to the INTEGRAL Burst Alert System (IBAS, Mereghetti et al. 2003a) providing near real-time, precise $\left(\sim 2^{\prime}-3^{\prime}\right)$ positioning of about one burst per month. This allows for follow-up observations of GRBs with the most sensitive facilities at different wavelengths. Here we report on the X-ray and optical/NIR studies of the afterglow

* Based on observations with XMM-Newton, an ESA science mission with instruments and contributions directly funded by ESA member states and the USA (NASA), and on observations collected at the European Southern Observatory, Paranal, Chile, under proposal 073.D-0255. of GRB 040827 as seen by the XMM-Newton observatory and the ESO Very Large Telescope (VLT), respectively.

\section{GRB 040827}

GRB 040827 was discovered by the INTEGRAL Burst Alert System on 2004, August 27 at 11:50:48 UT. The burst, detected with the IBIS/ISGRI instrument in the 15-200 keV band at $\alpha=15^{\mathrm{h}} 16^{\mathrm{m}} 59.8^{\mathrm{s}}, \delta=-16^{\circ} 08^{\prime} 21^{\prime \prime}$, with an uncertainty of 2.5', had a duration of $40 \mathrm{~s}$ (Mereghetti et al. 2004a,b). The peak flux in the $20-200 \mathrm{keV}$ band was estimated to be 0.6 photons $\mathrm{cm}^{-2} \mathrm{~s}^{-1}$, corresponding to $6.0 \times 10^{-8} \mathrm{erg} \mathrm{cm}^{-2} \mathrm{~s}^{-1}$ (Götz \& Mereghetti 2004).

An XMM-Newton observation of the field of GRB 040827 started on August 27 at 18:07:56 UT, 6 h 18 min after the burst. Soon after the beginning of the observation, analysis of realtime data showed an X-ray source (XMMU J151701.3-160828, Rodriguez-Pascual \& Gonzalez-Riestra 2004) within the INTEGRAL error circle, with an offset of $\sim 1$ arcmin with respect to the nominal aimpoint ${ }^{1}$. The telescope was therefore

\footnotetext{
1 See http://xmm.vilspa.esa.es/external/xmm_news/ items/grb040827/index.shtml
} 
re-pointed $\sim 4300 \mathrm{~s}$ after the beginning of the observation. The overall observing time was of $53.2 \mathrm{ks}$. The source XMMU J151701.3-160828 was found to be variable (Rodriguez-Pascual \& Juarez 2004), and therefore likely associated with the afterglow of GRB 040827. Such an identification was strengthened by the discovery of a fading NIR transient (see below).

Optical imaging of the INTEGRAL error box started immediately after the IBAS alert. Gladders \& Berger (2004) and Tanvir et al. (2004a) reported the presence inside the XMM-Newton error circle of a faint, extended source with $K_{\mathrm{S}} \sim 19.4$, named XMM 2, which was subsequently found to be variable (Kaplan \& Berger 2004; Tanvir et al. 2004b; Malesani et al. 2004a). No radio emission was detected from the source, with a $2 \sigma$ upper limit of $70 \mu \mathrm{Jy}, 4.5$ days after the GRB (Soderberg \& Frail 2004).

A preliminary report of the XMM-Newton observation was given by De Luca et al. (2004), but the results were affected by problems in the data possibly due to the satellite re-pointing. An improved attitude solution was then made available by the XMM-Newton SOC (Schartel 2004). We present here a detailed study of the X-ray dataset, coupled to an analysis of the complete set of observations performed with the VLT.

\section{XMM-Newton data analysis and results}

We have analyzed data collected by the European Photon Imaging Camera (EPIC). It consists of a PN detector (Strüder et al. 2001), with a collecting area of $\sim 1200 \mathrm{~cm}^{2}$ at $1.5 \mathrm{keV}$, and of 2 MOS detectors (Turner et al. 2001), with a collecting area of $\sim 450 \mathrm{~cm}^{2}$ each at $1.5 \mathrm{keV}$. All the cameras were operated in their full frame mode (providing imaging across the full $15^{\prime}$ radius field of view, with a time resolution of $73 \mathrm{~ms}$ in the PN and $2.6 \mathrm{~s}$ in the MOS), with the thin optical filter.

The Observation Data Files (ODFs) were retrieved from the XMM-Newton Science Archive and were processed with the XMM-Newton Science Analysis Software (XMM-SASv6.0.0), using standard pipeline tasks (epchain and emchain for the PN and the MOS, respectively) to produce calibrated photon lists, with reconstructed energy, time of arrival and coordinates on the field of view for each detected event.

The X-ray afterglow of GRB 040827 is clearly detected in all the cameras. Cross-correlating several field sources' positions with the USNO-B1 catalog improved the EPIC astrometry and yielded a source position at $\alpha=15^{\mathrm{h}} 17^{\mathrm{m}} 01^{\mathrm{s}} .46$, $\delta=-16^{\circ} 08^{\prime} 27^{\prime \prime}$. 2 , with a $1 \sigma$ uncertainty of $1^{\prime \prime} .5$. The position is fully consistent with that of the fading NIR afterglow.

The surface brightness radial profile of the source, computed for each EPIC detector, was fitted with a King function. The best fitting parameters were found to be consistent with the instrumental Point Spread Function (Read 2004). A claim of possible diffuse emission based on quick look data analysis (Rodriguez-Pascual \& Gonzalez-Riestra 2004) was clearly ascribable to problems in the events' attitude correction.

Source photons were extracted from a circle of 35 arcsec radius, containing $\gtrsim 85 \%$ of the events. Background events were selected from source-free regions of the same CCD chip where the target is imaged, following standard prescriptions from the

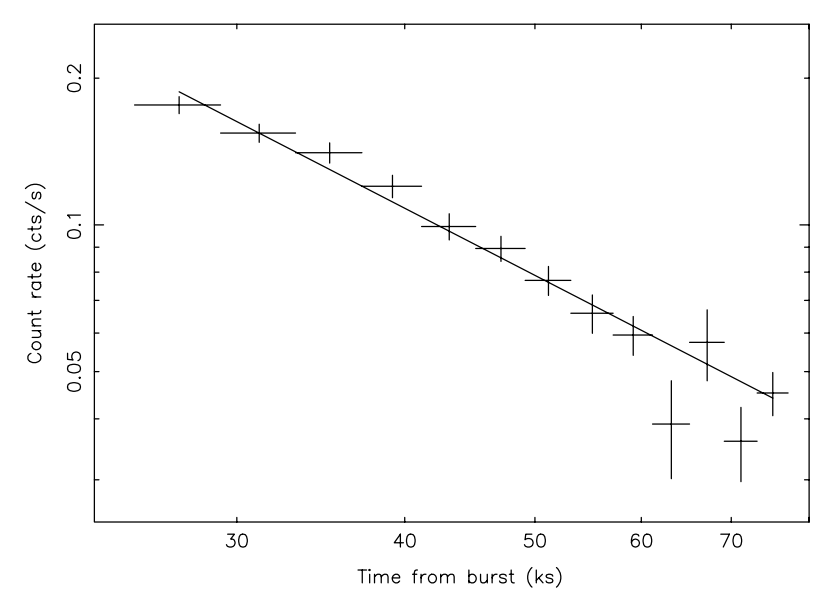

Fig. 1. Background-subtracted EPIC light curve $(0.2-10 \mathrm{keV})$ of the afterglow of GRB 040827. Data have been fitted with a power law decay $F \propto t^{-\delta}$, with a best fit value of $\delta=1.41 \pm 0.10\left(\chi_{v}^{2}=1.45\right.$, 11 d.o.f.).

calibration team (Kirsch 2004): for the PN we used a circle of 50 arcsec radius, located at the same distance from the readout node as the source region; for the MOS we used an annulus centered on the source position with inner and outer radii of 90 and 200 arcsec.

The source is clearly seen to fade during the observation. The background-subtracted light curve extracted from combined MOS and PN data is shown in Fig. 1. The time evolution of the observed count rate is consistent with a power law decay, $F \propto t^{-\delta}$, with $\delta=1.41 \pm 0.10\left(\chi_{v}^{2}=1.45,11\right.$ d.o.f. $)$. A somewhat better description $\left(\chi_{v}^{2}=0.79,9\right.$ d.o.f. $)$ of the decay is obtained with a broken power law model, with $\delta_{1}=0.9_{-0.5}^{+0.3}$, $\delta_{2}=1.67_{-0.20}^{+0.25}$ and break time $t_{\mathrm{b}}=36 \pm 6 \mathrm{ks}$ after the burst. With a simple F-test we evaluated the fit improvement to be significant at $\sim 97.3 \%$ level (well below $3 \sigma$ ).

In order to perform the spectral analysis, we screened data from time intervals affected by high particle background episodes (soft proton flares), obtaining good exposure times of $30.0 \mathrm{ks}, 38.8 \mathrm{ks}$ and $37.3 \mathrm{ks}$ for the PN, MOS1 and MOS2 cameras, respectively. Spectra for both source and background were extracted from the regions described above. Source spectra were rebinned in order to have at least 25 counts per channel. Ad-hoc response matrices and effective area files were computed with the SAS tasks rmfgen and arfgen ${ }^{2}$.

The spectral analysis was performed using XSPEC v11.3. Spectra from the three detectors were fitted simultaneously in the range $0.2-9 \mathrm{keV}$. All the errors on spectral parameters reported in this section are at $90 \%$ confidence level for a single interesting parameter.

An absorbed power law model (i.e. $\mathrm{d} N / \mathrm{d} E=$ $\mathrm{e}^{-\sigma(E) N_{\mathrm{H}}} K\left(E / E_{0}\right)^{\Gamma}$, where the photoelectric cross sections $\sigma(E)$ are from Balucinska-Church \& McCammon (1992), with updated He cross-sections, and $E_{0}=1 \mathrm{keV}$ )

\footnotetext{
2 Possibly owing to the $\sim 1^{\prime}$ change in pointing direction during the observation, to generate the effective area file it was necessary to describe the source region (in detector coordinates) using a larger number of bins than in standard cases (namely, arfgen parameters detxbins and detybins were set to 30 wrt. a default value of 5).
} 


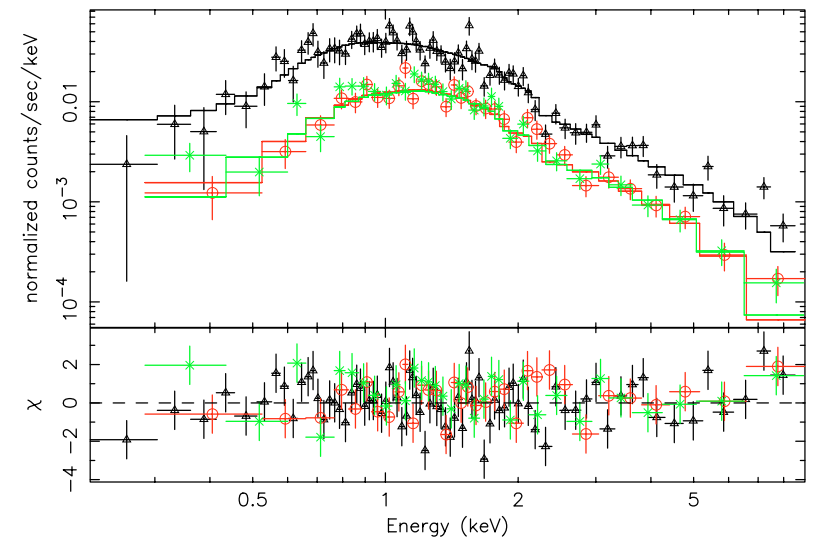

Fig. 2. EPIC observed spectra plotted together with the best fitting model convolved with the instrumental responses (upper panel); residuals in units of statistical standard errors (lower panel). Triangles, circles and crosses represent data from the PN, MOS1 and MOS2 cameras, respectively.

yields a reduced $\chi_{v}^{2}=1.35$ (145 d.o.f.), with photon index $\Gamma=2.4 \pm 0.1$ and column density $N_{\mathrm{H}}=(3.7 \pm 0.2) \times 10^{21} \mathrm{~cm}^{-2}$. Since the expected Galactic absorption in this direction varies from $\sim 5 \times 10^{20} \mathrm{~cm}^{-2}$ (Schlegel et al. 1998) to $\sim 8 \times 10^{20} \mathrm{~cm}^{-2}$ (Dickey \& Lockman 1990), we conclude that our best fitting $N_{\mathrm{H}}$ value is significantly higher than the Galactic one.

This is confirmed by the study of the brightest serendipitous source in the field, located at $\sim 7^{\prime}$ from the afterglow. Its spectrum is well fitted by an absorbed power law with $\Gamma=2.2 \pm 0.2$ and $N_{\mathrm{H}}=(9 \pm 3) \times 10^{20} \mathrm{~cm}^{-2}$. The position of such source was cross-correlated with multiwavelength catalogs, yielding (within $\sim 1^{\prime \prime}$ ) a USNO-B1 source with a magnitude $R \sim 19.3$, corresponding to a ratio $F_{\mathrm{X}} / F_{\text {opt }} \sim 8$, in the typical range of active galactic nuclei (Krautter et al. 1999). Moreover, a coincident radio source (NVSS J151717-160242) was found in the NED database. We conclude that such source is very likely an AGN and that the observed X-ray absorption is a reliable and independent estimate of the Galactic $N_{\mathrm{H}}$ in this direction.

On the other hand, the Galactic value of the column density is totally incompatible with the afterglow spectrum: forcing the $N_{\mathrm{H}}$ value to $8 \times 10^{20} \mathrm{~cm}^{-2}$ we obtain $\chi_{v}^{2}$ of 3.86 (146 d.o.f.).

The best description of the afterglow data was obtained by adding to the Galactic $N_{\mathrm{H}}\left(8 \times 10^{20} \mathrm{~cm}^{-2}\right)$ a redshifted neutral absorption component, $N_{\mathrm{H}, z}$. In this case the spectral shape is described as $\mathrm{d} N / \mathrm{d} E=\mathrm{e}^{-\sigma(E) N_{\mathrm{H}}} \mathrm{e}^{-\sigma[E(1+z)] N_{\mathrm{H}, z}} K\left(E / E_{0}\right)^{\Gamma}$, where $N_{\mathrm{H}}$ is fixed to the Galactic value of $8 \times 10^{20} \mathrm{~cm}^{-2}$ and $N_{\mathrm{H}, z}$ represents the gas column density in the host. In the redshift interval $0<z<3$, we obtained the best fit $\left(\chi_{v}^{2}\right.$ of 1.18 for 144 d.o.f. - see Fig. 2) for an intrinsic absorbing column $N_{\mathrm{H}, z} \sim 1.0 \times 10^{22} \mathrm{~cm}^{-2}$ at a redshift $z \sim 0.9$, and a power law photon index $\Gamma=2.3 \pm 0.1$. The observed $0.2-10 \mathrm{keV}$ flux is $\sim 2.2 \times 10^{-13} \mathrm{erg} \mathrm{cm}^{-2} \mathrm{~s}^{-1}$; the corresponding unabsorbed flux is $\sim 4.9 \times 10^{-13} \mathrm{erg} \mathrm{cm}^{-2} \mathrm{~s}^{-1}$.

Both $N_{\mathrm{H}, z}$ and $z$ are not well constrained towards large values, owing to their strong correlation. The contour plot for the redshifted absorber column density $N_{\mathrm{H}, z}$ vs. the redshift $z$, shown in Fig. 3, allows us to estimate that $N_{\mathrm{H}, z}>4 \times 10^{21} \mathrm{~cm}^{-2}$

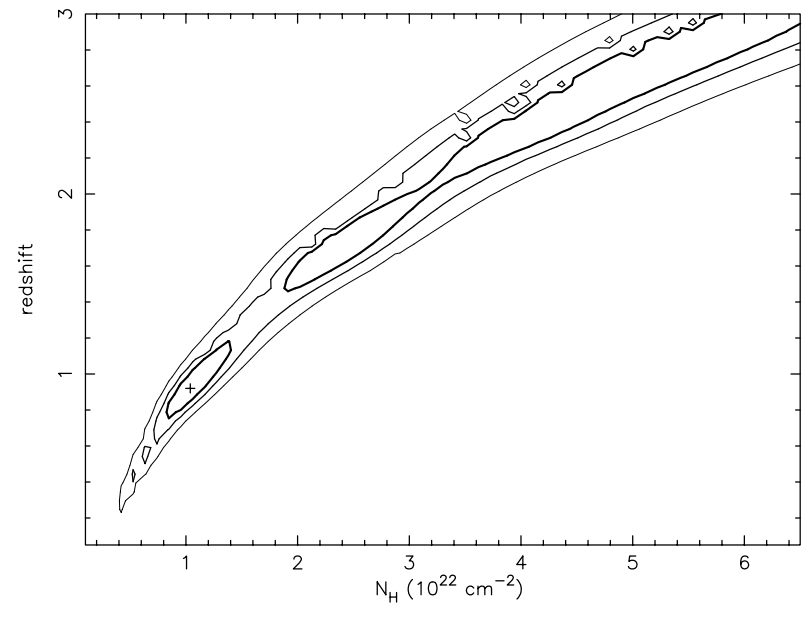

Fig. 3. Confidence contours $(68 \%, 90 \%$ and $99 \%)$ for the redshift and the column density of the redshifted cold absorber derived from the fit to EPIC spectra.

Table 1. Significance of additional $N_{\mathrm{H}}$. The F-test was used to compare different models for the X-ray absorption: $N_{\mathrm{H}}$ fixed to the Galactic value ("Gal. $N_{\mathrm{H}}$ "); $N_{\mathrm{H}}$ allowed to vary ("free $N_{\mathrm{H}}$ "); redshifted absorber added to the fixed Galactic $N_{\mathrm{H}}$ (" $N_{\mathrm{H}, z}$ "). The value of $F$ and the probability of a chance occurrence of the fit improvement are reported. The high significance of the extra absorption is apparent also in the pessimistic assumption of an actual Galactic $N_{\mathrm{H}} 50 \%$ higher than expected following Dickey \& Lockman (1990).

\begin{tabular}{ccc}
\hline \hline Absorption models & $F$ value & $P(F)$ \\
\hline Gal. $N_{\mathrm{H}}$ vs. free $N_{\mathrm{H}}$ & 274 & $3.0 \times 10^{-35}$ \\
Gal. $N_{\mathrm{H}}$ vs. $N_{\mathrm{H}, z}$ & 167 & $2.8 \times 10^{-38}$ \\
\hline Gal. $N_{\mathrm{H}}+50 \%$ vs. free $N_{\mathrm{H}}$ & 187 & $6.8 \times 10^{-28}$ \\
Gal. $N_{\mathrm{H}}+50 \%$ vs. $N_{\mathrm{H}, z}$ & 118 & $5.3 \times 10^{-31}$ \\
\hline Free $N_{\mathrm{H}}$ vs. $N_{\mathrm{H}, z}$ & 21.5 & $8.0 \times 10^{-6}$ \\
\hline
\end{tabular}

and $z>0.5$ at $90 \%$ confidence level (for two parameters of interest).

To evaluate the statistical significance of the additional $N_{\mathrm{H}, z}$, we used the F-test, comparing the models with: (i) $N_{\mathrm{H}}$ fixed to the Galactic value; (ii) $N_{\mathrm{H}}$ allowed to vary; (iii) redshifted absorber added to the fixed Galactic $N_{\mathrm{H}}$. Results are reported in Table 1. The high significance of the intrinsic absorption is evident even in the pessimistic assumption of a Galactic $N_{\mathrm{H}} 50 \%$ higher than expected, a value which could be marginally consistent with the absorption seen in the spectrum of the serendipitous AGN.

We note that the residuals plotted in Fig. 2 (lower panel) show several small wiggles. In order to search for possible emission lines in the afterglow's spectrum, we tried to add gaussian lines, both single and multiple (up to 5 at a time). The width was fixed (smaller than the instrumental energy resolution); the central energy, together with the line flux, was allowed to vary in the range $0.5-5 \mathrm{keV}$, where most of the statistics are collected. No significant lines were detected in such range in the combined MOS/PN dataset. The upper limits $(3 \sigma)$ on the equivalent width of any emission line are $\sim 60 \mathrm{eV}$ and $\sim 250 \mathrm{eV}$ in the ranges $0.5-2$ and $2-5 \mathrm{keV}$, respectively. 
Table 2. GRB 040827. Observation log. Observing dates are referred to the middle of the exposures.

\begin{tabular}{lllclll}
\hline \hline $\begin{array}{l}\text { Date } \\
\text { (UT) }\end{array}$ & $\begin{array}{l}\text { Time since burst } \\
(\text { days })\end{array}$ & $\begin{array}{l}\text { Filter } \\
\text { 2004 Aug. 29.99 }\end{array}$ & $\begin{array}{c}\text { Exposure time } \\
(\mathrm{s})\end{array}$ & $\begin{array}{l}\text { Seeing } \\
\left({ }^{\prime \prime}\right)\end{array}$ & $\begin{array}{l}\text { Instrument } \\
\text { Magnitude }\end{array}$ \\
2004 Aug. 30.04 & 2.54 & $R$ & $5 \times 120$ & 1.2 & VLT+FORS 1 & $23.55 \pm 0.50$ \\
\hline 2004 Aug. 28.99 & 1.50 & $R$ & $10 \times 120$ & 0.6 & VLT+FORS 2 & $23.02 \pm 0.15$ \\
2004 Aug. 30.01 & 2.52 & $I$ & $4 \times 180$ & 1.4 & VLT+FORS 1 & $22.47 \pm 0.15$ \\
2004 Sep. 01.99 & 5.51 & $I$ & $5 \times 240$ & 0.5 & VLT+FORS 2 & $22.64 \pm 0.13$ \\
2004 Sep. 09.01 & 12.51 & $I$ & $10 \times 180$ & 0.6 & VLT+FORS 2 & $22.61 \pm 0.10$ \\
& & $I$ & $6 \times 180$ & 0.4 & VLT+FORS 2 & $22.33 \pm 0.20$ \\
2004 Sep. 16.99 & 20.50 & & & & & \\
\hline 2004 Aug. 28.06 & 0.57 & $I$ & $10 \times 30$ & 1.0 & VLT+ISAAC & $20.92 \pm 0.28$ \\
\hline 2004 Aug. 27.97 & 0.47 & $H$ & $10 \times 30$ & 0.8 & VLT+ISAAC & $19.42 \pm 0.13$ \\
\hline 2004 Aug. 27.96 & 0.47 & $K_{\mathrm{s}}$ & $10 \times 30$ & 0.7 & VLT+ISAAC & $19.02 \pm 0.30$ \\
2004 Aug. 28.01 & 0.51 & $K_{\mathrm{s}}$ & $10 \times 30$ & 0.9 & VLT+ISAAC & $18.94 \pm 0.10$ \\
2004 Aug. 28.98 & 1.48 & $K_{\mathrm{s}}$ & $60 \times 30$ & 0.9 & VLT+ISAAC & $20.05 \pm 0.13$ \\
2004 Sep. 16.02 & 19.52 & $K_{\mathrm{s}}$ & $30 \times 60$ & 1.0 & VLT+ISAAC & $20.08 \pm 0.15$ \\
2004 Sep. 24.01 & 27.52 & $K_{\mathrm{s}}$ & $45 \times 60$ & 0.6 & VLT+ISAAC & $19.88 \pm 0.10$ \\
2004 Sep. 28.00 & 31.50 & $K_{\mathrm{s}}$ & $48 \times 60$ & 1.2 & VLT+ISAAC & $20.35 \pm 0.20$ \\
\hline 2004 Aug. 30.05 & 2.54 & $300 \mathrm{~V}$ & $4 \times 1800$ & 1.1 & VLT+FORS 1 & - \\
\hline
\end{tabular}

Thermal models (requiring, in any case, extra absorption wrt. the Galactic $N_{\mathrm{H}}$ ) cannot fit the data equally well. A redshifted, optically thin plasma model (MEKAL in XSPEC), with $z$ linked to the redshift of the neutral cold absorber $N_{\mathrm{H}, z}$, yields rather poor results ( $\chi_{v}^{2}$ of 1.89 for 144 d.o.f.) when abundances are fixed to solar system values. If abundances are allowed to vary, better results can be obtained. However, the resulting $\chi_{v}^{2}$ is worse $(>1.4)$ than for the simple power law model.

We tried also a composite model encompassing a power law as well as a plasma emission model, but the resulting $\chi^{2}$ is similar to that obtained using the simpler power law model.

We searched for a possible spectral evolution, using the power law plus redshifted absorber model. We divided the observation in two time intervals of $\sim 13$ and $\sim 22 \mathrm{ks}$, in order to have approximately the same number of source photons in each subset, and extracted the corresponding spectra for each EPIC detector. In a simultaneous fit, a simple variation of the power law intensity yields a good description of the data $\left(\chi_{v}^{2}\right.$ of 1.0 for 180 d.o.f.). No significant evolution is seen for the absorbing column $N_{\mathrm{H}, z}$ and the photon index. No significant deviations from the smooth continuum are seen in the time-resolved data.

\section{VLT observations}

Near-infrared (NIR) observations of the error box of GRB 040827 started as soon as possible after the notification of the trigger. We used the ISAAC camera installed at the Nasmyth focus of the ESO VLT-UT1 (Antu) located at Paranal (Chile). Since the field of view of ISAAC $\left(2.5^{\prime} \times 2.5^{\prime}\right)$ is not large enough to image the whole INTEGRAL error box, a mosaic of four exposures was obtained. In the following nights, further optical and NIR observations were secured (Table 2). Optical data were acquired with the FORS 1 and FORS 2 instruments mounted at the Cassegrain focus of the VLT-UT2 and UT1 respectively. Data reduction was performed using the standard procedures. Both aperture and profile photometry (using DAOPHOT - Stetson 1987) were adopted to extract instrumental magnitudes. Absolute calibration was achieved by observing standard fields (in the optical) and by using the 2MASS catalog (in the infrared). The consistency of the zeropoint was checked in different nights. In some cases the photometric errors are larger than usual, since the object could be observed just for a small part of the night, at a large airmass.

The source reported by Gladders \& Berger (2004) and Tanvir et al. (2004a) was clearly detected in our images at the coordinates $\alpha=15^{\mathrm{h}} 17^{\mathrm{m}} 01^{\mathrm{s}} .34, \delta=$ $-16^{\circ} 08^{\prime} 29^{\prime}$. 1 (J2000, $\sim 0.2^{\prime \prime}$ uncertainty, based on 9 nonsaturated USNO stars). Its magnitude was $K_{\mathrm{s}}=18.95 \pm 0.08$ on Aug. 27.98 (average of our first two measurements), and it showed a dimming by $1.10 \pm 0.15 \mathrm{mag}$ in the $K_{\mathrm{s}}$ filter, between 2004 Aug. 27.98 and 28.98 (0.49 and 1.48 days after the GRB, respectively), thus confirming the reported variation. Since the seeing was similar in the two epochs $\left(\approx 0.9^{\prime \prime}\right)$, this result is robust even if in our best-seeing images there are hints that the object is slightly extended (with a size of $\sim 0.5^{\prime \prime}$ ).

Long-term monitoring was performed in the $I$ and $K_{\mathrm{s}}$ bands (see Fig. 4). No significant variation of the source was seen from 1.5 up to $\sim 30$ days after the burst, indicating that the source was dominated by the host galaxy. This is consistent with the source being extended. In particular, there is no sign of afterglow emission in the $R$ and $I$ bands (for which we have 


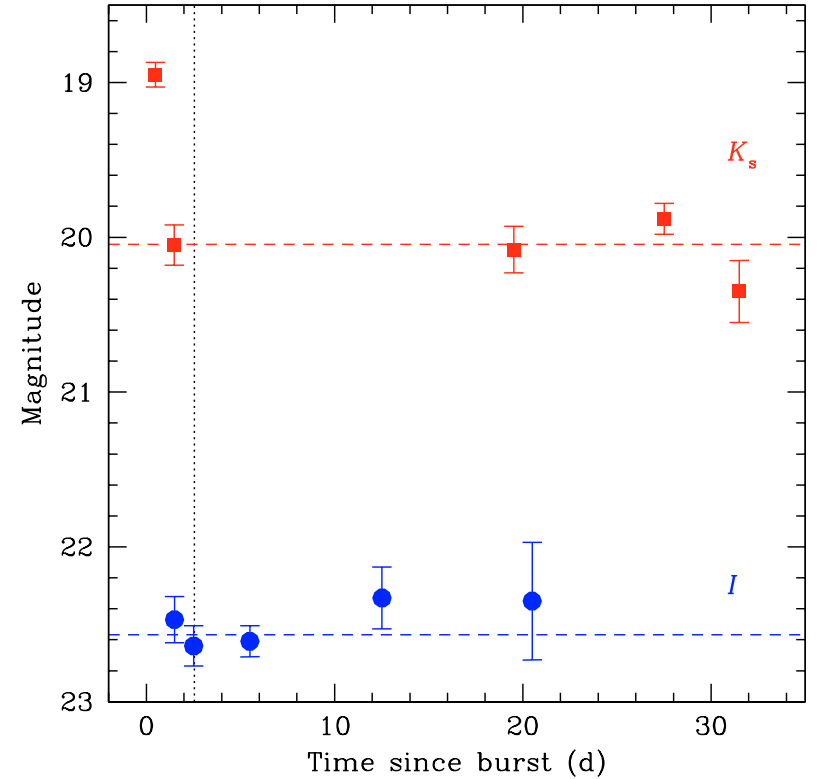

Fig. 4. Light curve of the optical counterpart of GRB 040827. Squares and circles correspond to $K_{\mathrm{s}}$ - and $I$-band measurements respectively. The first $K_{\mathrm{s}}$-band point is the average of our first two exposures. The horizontal dashed lines show the host galaxy contribution, and the vertical dotted line marks the epoch of our spectrum.

not data during the first night). In our early $J$ and $H$ images, a contribution from the afterglow is likely present, but the host galaxy is contributing by a significant, unknown amount, hampering the determination of the intrinsic afterglow brightness. After averaging over the available measurements, we get for the host galaxy $R=23.10 \pm 0.12, I=22.57 \pm 0.06$ and $K_{\mathrm{s}}=20.05 \pm$ 0.07 . Therefore, we can estimate the afterglow magnitude to be $K_{\mathrm{s}}=19.44 \pm 0.13$ and $K_{\mathrm{s}}>21.02(3 \sigma)$ on 2004 Aug. 27.98 and 2004 Aug. 28.97 respectively. Assuming a power law decay $\left(F(t) \propto t^{-\delta}\right)$, the decay index is constrained to be $\delta>$ $1.30(3 \sigma)$. We caution that this value is computed from just two measurements, and may be influenced by local deviations from the regular decay and/or measurement errors. This limit is consistent with the X-ray decay index.

A spectrum of the host galaxy was acquired with FORS 1, starting on 2004 Aug. 30.00 (2 h exposure with the grism $300 \mathrm{~V})$. The observing conditions were not good, since the object was visible only at relatively large airmass $(\sim 2)$ and with full $(100 \%)$ Moon. The object was marginally detected in the range $4600 \div 8600 \AA$, characterized by a very weak continuum with no distinguishable features. Therefore, no redshift determination was possible. The 2- $\sigma$ upper limit for any emission line is $10^{-16} \mathrm{erg} \mathrm{cm}^{-2} \mathrm{~s}^{-1}$.

Costraints on the distance of the galaxy can be put using the photometric information. With just two colors, photometric redshift techniques are not very effective. The algorithm developed by Fernández-Soto at al. (1999) yields just loose constraints $1.0<z<3.5$, with an additional permitted range $0.1<z<0.3$ (where the fit is worse).

However, we can use the apparent luminosity to get a rough estimate of the distance. To this extent, we have chosen several catalogs of galaxies providing for each object both the photometry and the redshift. From these data, we computed the redshift distribution of the sources that have a magnitude comparable to that of the host galaxy of GRB 040827. Data were collected from the VIMOS VLT deep survey (VVDS; Le Fèvre et al. 2004), the Hubble deep field (Fernández-Soto at al. 1999), the FORS deep field (Heidt et al. 2003; Noll et al. 2004) and the Caltech faint galaxy redshift survey (Cohen et al. 2000). We found that in the $K_{\mathrm{s}}$ band the distribution is quite broad (peaking at $z=0.9$ ), and it does not allow to determine significant contraints. Tighter limits can be set by looking at the I-band apparent luminosity; the distribution of 127 galaxies from the VVDS within $0.15 \mathrm{mag}$ from the GRB host is well represented by a Gaussian centered at $z=0.77$ with a dispersion of 0.31 . The VVDS is particularly suited for this kind of work, since it has a high completeness in redshift determination, and because it succesfully crosses the so-called redshift desert $1.5<z<2.7$ (Le Fèvre et al. 2004), where redshift determination is difficult due to the lack of clear features. Thus, based on a statistical analysis, we may put a $3 \sigma$ upper limit $z<1.7$. The same procedure in the $R$ band provides an even narrower range $(z=0.66 \pm 0.23)$, however based on a smaller sample. We caution that our object, since it hosted a GRB, may not be a typical galaxy, therefore our indicator may be biased. It is anyway reassuring that the estimate from both the $R$ and $I$ bands are consistent. Thus, the different indications coming from both optical and X-ray data favor a redshift not far from unity. This is consistent with the redshift distribution of GRBs (e.g. Bloom et al. 2003).

Within the photometric errors, no late-time rebrightening is apparent in our data. However, even a bright supernova like SN 2003lw (Malesani et al. 2004b) peaked at $J \sim 18.8$ (at $z=$ 0.1055 ), which would translate to $K_{\mathrm{s}} \sim 22.8$ at $z \sim 1$ (the observed $K_{\mathrm{s}}$ band roughly corresponds to the $J$ at this redshift). Such a SN would be $\sim 13$ times fainter than the host galaxy, hence quite difficult to detect.

\section{Discussion}

XMM-Newton/EPIC data show unambiguously that the spectrum of the X-ray afterglow is affected by an absorption significantly higher than the Galactic value.

As shown in Sect. 3, assuming solar system abundances, the redshifted absorber model allows us to estimate that at $90 \%$ confidence the local column density in the GRB host $N_{\mathrm{H}, z}$ is higher than $4 \times 10^{21} \mathrm{~cm}^{-2}$ and its redshift is larger than 0.5 . Furthermore, optical photometry (Sect. 4) allows us to estimate that the host redshift is likely lower than 1.7. When such a constraint is used, it is possible to limit the allowed interval for the intrinsic $N_{\mathrm{H}, z}$ to the range $(0.4-2.6) \times 10^{22} \mathrm{~cm}^{-2}$.

We investigated the possible presence of optical extinction in the host, coupled to the observed large X-ray absorption. First, we computed the spectral energy distribution of the afterglow, taking advantage of our almost simultaneous VLT $K_{\mathrm{s}}$ and XMM observations. The observed $K_{\mathrm{s}}$ band flux falls about two orders of magnitude below the extrapolation of the X-ray power law. Then, we followed the approach of Price et al. (2002) in order to get constraints on the expected spectral shape. To calculate the "Closure" relations, we assumed 
Table 3. Constraints on the fireball model. The observed spectral index and time decay index are used to test different afterglow expansion models: (i) a simple isotropic fireball expansion in an homogeneous interstellar medium ("ISM", Sari et al. 1998); (ii) an isotropic fireball expansion in a stellar wind density profile environment ("Wind", Chevalier \& Li 1999); (iii) expansion of a collimated flow ("Jet", Sari et al. 1999). We compute the "closure" relation (Price et al. 2002) $C=\delta+b \alpha+c$, where $\delta$ is the index of the temporal decay and $\alpha$ is the spectral index of the afterglow; the values of the parameters $b$ and $c$ depend on the model used (ISM, Wind, Jet). The cases of electron cooling frequency higher $\left(v_{\mathrm{c}}>v_{\mathrm{X}}\right)$ or lower $\left(v_{\mathrm{c}}<v_{\mathrm{X}}\right)$ than the EPIC range of sensitivity are considered. A specific model is consistent with the data if the Closure value is consistent with 0 .

\begin{tabular}{ccc}
\hline \hline Model & Closure $\left(v_{\mathrm{c}}<v_{\mathrm{X}}\right)$ & Closure $\left(v_{\mathrm{c}}>v_{\mathrm{X}}\right)$ \\
\hline ISM & $-0.04 \pm 0.18$ & $-0.54 \pm 0.18$ \\
Wind & $-0.04 \pm 0.18$ & $-1.04 \pm 0.18$ \\
Jet & $-1.19 \pm 0.22$ & $-2.19 \pm 0.22$ \\
\hline
\end{tabular}

the single power law decay described in Sect. 3. The NIR decay is loosely constrained and is compatible with the X-ray one. The observed properties of the afterglow are consistent with an isotropic fireball expansion both into an homogeneous medium, as well as into a stellar wind density profile environment (see Table 3 and its caption for further details). Both cases require the electron cooling frequency $v_{\mathrm{c}}$ to be lower than the X-ray range (namely, $v_{\mathrm{c}}<1.2 \times 10^{17} \mathrm{~Hz}$ ), a constraint which may be easily fulfilled at the time of the X-ray observation. Incidentally, we note that such results agree with the "typical" X-ray afterglow behaviour derived by Piro (2004) from 36 BeppoSAX follow-up observations of GRBs. The possible presence of a spectral break between the X-rays and the $K_{\mathrm{S}}$ band (if $v_{K}<v_{\mathrm{c}}<v_{\mathrm{X}}$ ) does not allow us to conclude that the optical flux is affected by a high extinction; of course, such a possibility may not be ruled out, since $v_{\mathrm{c}}$ could be lower than the NIR range. Both pictures can be easily accomodated within the constraints on the host redshift $(0.5<z<1.7)$ and gas column density $\left(0.4-2.6 \times 10^{22} \mathrm{~cm}^{-2}\right)$, using standard extinction curves (Cardelli et al. 1989), both assuming the Galactic gas-to-dust mass ratio (Predehl \& Schmitt 1995) and assuming different gas/dust properties in the host (implying an extinction up to 10 times lower, as suggested by several studies, e.g. Galama \& Wijers 2001; Hjorth et al. 2003; Stratta et al. 2004).

$\mathrm{X}$-ray absorption in excess relative to the Galactic one has been already reported for a handful of GRB afterglows. Thus, to put our results in the right context, it is useful to review here the results on X-ray absorption in GRB afterglows (observed a few hours after the burst) obtained so far by BeppoSAX, Chandra and XMM-Newton. Stratta et al. (2004) present a systematic analysis of a sample of 13 bright afterglows observed with BeppoSAX narrow field instruments (within 5-20 h of the prompt); a significant ( $>99.9 \%$ confidence) detection of additional $N_{\mathrm{H}}$ was found only in two cases (namely, GRB 990123 and GRB 010222), but, owing to the limited photon statistics, it could not be excluded that intrinsic X-ray absorption was present also in the other bursts. Chandra observations of GRB afterglows (starting, on average, $\gtrsim 1$ day after the GRB), have not yielded so far useful constraints on the presence of intrinsic X-ray absorption, apart from a marginal detection for GRB 020405 (see Stratta et al. 2004, and references therein).

To date, XMM-Newton has observed 9 GRB afterglows (including GRB 040827) within a few hours $(\sim 6 \div 15)$ of the burst $^{3}$. A detailed summary of XMM-Newton results is reported in Table 4 as well as in Fig. 5. Extra absorption has been found in 4 cases, namely GRB 020322, GRB 030227 , GRB 040223 and GRB 040827 (the best case in such a sample - note Fig. 5). On the contrary, absorption consistent with the Galactic one was observed for GRB 001025 , GRB 010220 , GRB 011211, GRB 031203 and GRB 040106 (the brightest afterglow observed by XMM-Newton so far).

No firm conclusions about the presence of significant, intrinsic optical extinction may be drawn for the X-ray "absorbed" afterglows. The case of GRB 020322 (Watson et al. 2002a; Gendre et al. 2005) is very similar to GRB 040827 . The lack of an optical afterglow for GRB 040223 (Simoncelli et al. 2004) could also be explained by intrinsic optical extinction within the same hypotheses (Gendre et al. 2005), although a large foreground extinction is possibly playing an important role in such burst, owing to its location, close to the Galactic plane. A complete optical dataset is available only for GRB 030227, including multicolor, multi-epoch photometry of the afterglow. Interestingly, in such a case Castro-Tirado et al. (2003) found it "not feasible" to connect the X-ray and optical flux distributions within the frame of standard afterglow models, even considering different extinction laws in the host; therefore, they invoke different emission mechanisms at optical and X-ray wavelengths.

In the X-ray "unabsorbed" sample, GRB 011221 (Jakobsson et al. 2003) and GRB 040106 (Gendre et al. 2004; Moran et al. 2005) show very little or no optical extinction. The case of GRB 031203 is more problematic: significant extinction is observed (Prochaska et al. 2004), although it is hard to discriminate the Galactic and host contributions. Moreover, Malesani et al. (2004b) show that the optical and the $\mathrm{X}$-ray emission of the afterglow may have a different origin. No optical emission was detected for the cases of GRB 001025 (for which the trigger was not promptly communicated; Smith et al. 2000) and GRB 010220 (also lying towards the Galactic plane).

Thus, X-ray data provide now conclusive evidence that several GRBs do occur behind a large gas column density in their host galaxy. If long GRBs are associated to the death of massive stars, indications of a large intrinsic $N_{\mathrm{H}}$ absorbing the X-ray afterglows' emission would imply that their progenitors are located within dense environments in their host galaxies, most probably star-forming regions (see e.g. Owens et al. 1998; Galama \& Wijers 2001). In the same scenario, the possible low extinction and reddening for the optical afterglows could be explained invoking dust destruction by the early radiation of the GRB itself, implying that the bulk of

\footnotetext{
${ }^{3}$ We exclude here the bright afterglow of GRB 030329, since it could be observed only two months after the burst (Tiengo et al. 2003), owing to satellite pointing constraints.
} 
Table 4. Summary of XMM-Newton observations of intrinsic gas column density in GRB hosts. Starting from the left, columns show (1) the GRB Id.; (2) the Galactic $N_{\mathrm{H}}\left(\mathrm{cm}^{-2}\right)$ in the GRB direction according to Dickey \& Lockman (1990); (3) the observed $N_{\mathrm{H}}\left(\mathrm{cm}^{-2}\right)$, assuming solar system abundances and a power law model for the afterglow (for GRB 001025, GRB 010220 and GRB 011211, $N_{\mathrm{H}}$ was simply fixed to the Galactic value, which yielded a satisfactory fit); (4) the allowed range of redshifts for the host ("abs": from X-ray spectroscopy using a redshifted absorber model; "lin": from X-ray spectroscopy, based on emission line detections; "opt": from optical spectroscopy); (5) the local gas column density $N_{\mathrm{H}, z}\left(\mathrm{~cm}^{-2}\right)$ at the fiducial host redshift reported between parentheses (the value $z=1$ was assumed when no redshift constraints are available). If not available in literature, $N_{\mathrm{H}, z}$ was computed simply scaling by $(1+z)^{2.6}$ the difference between observed and expected (Galactic) $N_{\mathrm{H}}$; (6) the reference publication: a - Watson et al. (2002a); b - Mereghetti et al. (2003b); c - Watson et al. (2003); d - Tiengo et al. (2004); e - Watson et al. (2002b); f - Reeves et al. (2002); g - Watson et al. (2004); h - Prochaska et al. (2004); i - Gendre et al. (2004).

\begin{tabular}{|c|c|c|c|c|c|}
\hline \multicolumn{6}{|c|}{ Absorbed afterglows } \\
\hline GRB & Galactic $N_{\mathrm{H}}$ & Observed $N_{\mathrm{H}}$ & $z$ range (method) & $N_{\mathrm{H}, z}(z)$ & Reference \\
\hline 020322 & $4.6 \times 10^{20}$ & $(2.1 \pm 0.3) \times 10^{21}$ & $0.7-2.8$ (abs) & $1.3 \times 10^{22}(1.8)$ & $\mathrm{a}$ \\
\hline 030227 & $1.8 \times 10^{21}$ & $(3.6 \pm 0.3) \times 10^{21}$ & $2.5-4.5(\mathrm{abs})$ & $6.8 \times 10^{22}(3.9)$ & $\mathrm{b}$ \\
\hline “ & “ & “ & $1.33-1.42$ (lin) & $3.5 \times 10^{22}(1.39)$ & $\mathrm{c}$ \\
\hline 040223 & $6.0 \times 10^{21}$ & $(1.8 \pm 0.2) \times 10^{22}$ & $\ldots$ & $7 \times 10^{22}(1)$ & $\mathrm{d}$ \\
\hline 040827 & $8 \times 10^{20}$ & $(3.7 \pm 0.2) \times 10^{21}$ & $0.5-1.7$ (abs, opt) & $1.0 \times 10^{22}(1)$ & this work \\
\hline \multicolumn{6}{|c|}{ Unabsorbed afterglows } \\
\hline GRB & Galactic $N_{\mathrm{H}}$ & Observed $N_{\mathrm{H}}$ & $z$ range (method) & & Reference \\
\hline 001025 & $6 \times 10^{20}$ & $=$ & $0.50-0.55$ (lin) & & $\mathrm{e}$ \\
\hline 010220 & $8.6 \times 10^{21}$ & $=$ & $0.97-1.07$ (lin) & & $\mathrm{e}$ \\
\hline 011211 & $4.2 \times 10^{20}$ & $=$ & 2.14 (opt) & & $\mathrm{f}$ \\
\hline 031203 & $5.9 \times 10^{21}$ & $(8.8 \pm 0.5) \times 10^{21}$ & 0.105 (opt) & & $\mathrm{g}, \mathrm{h}$ \\
\hline 040106 & $8.6 \times 10^{20}$ & $(8.8 \pm 0.8) \times 10^{20}$ & $\ldots$ & & $\mathrm{i}$ \\
\hline
\end{tabular}

the absorbing gas is confined in a compact cloud (10-30 pc) surrounding the GRB progenitor, as suggested by previous studies (see e.g. Galama \& Wijers 2001). Indeed, the gas column densities reported in Table 4 are fully consistent with values observed for inner, overdense regions of giant molecular clouds in our galaxy (see e.g. Lazzati \& Perna 2002, and references therein), where star formation is supposed to take place. The rather shallow constraints on the optical extinction are generally consistent with such a picture.

On the other hand, very little may be concluded about the sample of X-ray "unabsorbed" afterglows. Lack of statistics in their X-ray spectra coupled to the uncertainty on the expected Galactic absorption, together with the uncertainty affecting the host redshift (based in two cases on highly debated detections of X-ray emission lines) leave room for at least a few $10^{21} \mathrm{~cm}^{-2}$ of gas column density in their host galaxies. Moreover, such GRBs do not have any peculiar aspect of their phenomenology clearly distinguishing them from the "absorbed" sample. Thus, apparently there are no reasons to invoke a different origin for the "unabsorbed" GRBs.

Alternatively, the intrinsic X-ray absorption could be mainly related to the large scale gas distribution in the GRB host. In this case, the X-ray phenomenology of the afterglow would depend on the location of the progenitor within the galaxy, on the structure of the host galaxy and on its orientation relative to the observer, making it easier to account both for absorbed as well as unabsorbed afterglows. Under this hypothesis the low optical extinction and reddening could be explained by different dust-to-gas mass ratios and/or low metallicities in the GRB hosts than in our Galaxy (as suggested by different investigations, e.g. Hjorth et al. 2003; Stratta et al. 2004).

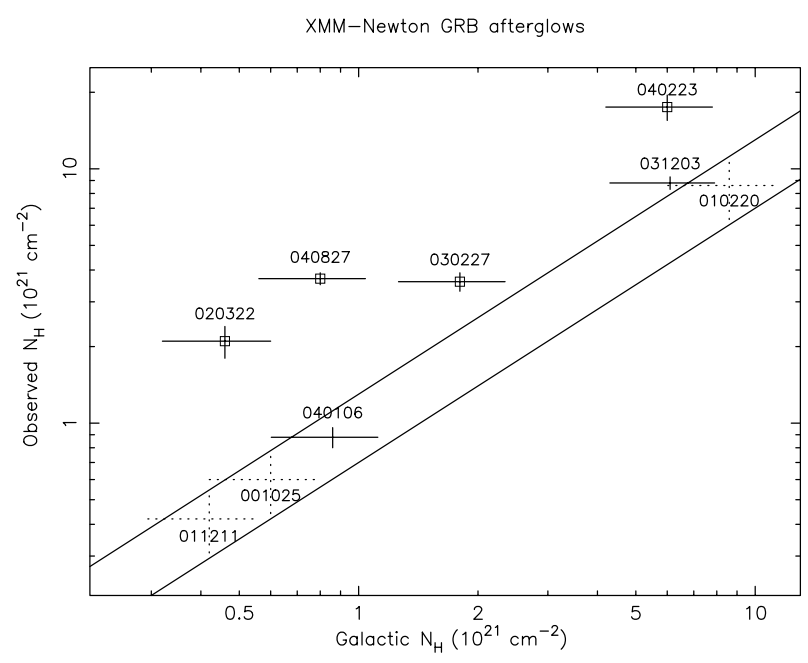

Fig. 5. Summary of XMM-Newton results on intrinsic absorption in GRB afterglows. Solid lines correspond to an $N_{\mathrm{H}}=$ Galactic $N_{\mathrm{H}} \pm 30 \%$, which represent a reasonable guess (see e.g. Stratta et al. 2004) of the uncertainty affecting Galactic column density estimates. "Absorbed afterglows" are marked with a hollow square. For GRB 001025, GRB 010220 and GRB 011211 the $N_{\mathrm{H}}$ value was not evaluated with spectral fitting, but just fixed to the Galactic value, yielding satisfactory results. We attached to such points an error bar (in dotted style) corresponding to $30 \%$ of the $N_{\mathrm{H}}$. Reference publications are listed in the caption to Table 4 .

We note that the problem of inferring properties of the inner GRB environment from the afterglow's absorption may be further complicated by the effects of photo-ionization by the GRB radiation itself (Lazzati \& Perna 2002); some evidence 
for such a process has been obtained from prompt soft X-ray observations with BeppoSAX (e.g. Frontera et al. 2004, and references therein).

\section{Conclusions}

Afterglow emission from GRB 040827 has been studied with XMM-Newton and with the VLT.

The observed spectral and temporal properties, when considered in the framework of different fireball models, are consistent with isotropic expansion into an homogeneous medium, or into a stellar-wind density profile environment. This is a somewhat typical behaviour for X-ray afterglows.

On the other hand, an outstanding peculiarity of GRB 040827 is the presence of very high intrinsic X-ray absorption, the best case observed so far, which may be attributed to a gas column density of $(0.4-2.6) \times 10^{22} \mathrm{~cm}^{-2}$ in the host galaxy, likely located at $0.5<z<1$.7. Intrinsic absorption is possibly not a ubiquitous property of GRB X-ray afterglows, since it affects $\sim 50 \%$ of the XMM-Newton afterglow observations. The presence of high optical extinction accompanying the observed X-ray absorption cannot be firmly proved, either for GRB 040827 or for other X-ray absorbed afterglows.

Current X-ray observations do not allow us to understand the nature of the ambient medium surrounding GRB progenitors. A major step forward could come only from a study of the evolution of the X-ray absorption (coupled to optical extinction) in the prompt emission and in the very early phases of the afterglow for a relatively large sample of events. This is now possible with the Swift satellite (Gehrels et al. 2004), thanks to its unprecedented capabilities of re-pointing its X-ray and optical telescopes in a few tens of seconds.

Acknowledgements. The XMM-Newton data analysis is supported by the Italian Space Agency (ASI). A.D.L. acknowledges an ASI fellowship. A.F.S. acknowledges support from projects AYA2002-03326 and GV/GRUPOS03/170.

\section{References}

Balucinska-Church, M., \& McCammon, D. 1992, ApJ, 400, 699 Bloom, J. S., Djorgowski, S. G., \& Kulkarni, S. R. 2001, ApJ, 554, 678

Bloom, J. S., Frail, D. A., \& Kulkarni, S. R. 2003, ApJ, 594, 674

Cardelli, J. A., Clayton, G. C., \& Mathis, J. S. 1989, A\&A, 345, 245

Castro-Tirado, A. J., Gorosabel, J., Guziy, S., et al. 2003, A\&A, 411, L315

Chevalier, R. A., \& Li, Z.-Y. 1999, ApJ, 520, L29

Cohen, J. G., Hogg, D. W., Blandford, R., et al. 2000, ApJ, 538, 29

De Luca, A., Götz, D., \& Campana, S. 2004, GCN, 2698

Dickey, J. M., \& Lockman, F. J. 1990, ARA\&A, 28, 215

Fernández-Soto, A., Lanzetta, K. M., \& Yahil, A. 1999, ApJ, 513, 34

Frontera, F., Amati, L., Lazzati, D., et al. 2004, ApJ, 614, 301

Fynbo, J. P. U., Jakobsson, P., Möller, P., et al. 2003, A\&A, 406, L63

Galama, T. J., \& Wijers, R. A. M. J. 2001, ApJ, 549, L209

Gehrels, N., Chincarini, G., Giommi, P., et al. 2004, ApJ, 611, 1005

Gendre, B., Piro, L., \& De Pasquale, M. 2004, A\&A, 424, L27

Gendre, B., Piro, L., \& De Pasquale, M. 2005, Adv. Sp. Res., in press [arXiv: astro-ph/0412302]

Gladders, M., \& Berger, E. 2004, GCN, 2681
Götz, D., \& Mereghetti, S. 2004, GCN, 2671

Heidt, J., Appenzeller, I., Gabasch, A., et al. 2003, A\&A, 398, 49

Hjorth, J., Moller, P., Gorosabel, J., et al., 2003, ApJ, 597, 699

Hurley, K., Sari, R., \& Djorgowski, S. G. 2003, in Compact Stellar $\mathrm{X}$-ray Sources (Cambridge University Press) [arXiv: astro-ph/0211620]

Jakobsson, P., Hjorth, J., Fynbo, J. P. U., et al. 2003, A\&A, 408, 941

Kaplan, D. L., Berger, E. 2004, GCN, 2683

Kirsch, M. (on behalf of the calibration team), EPIC status of calibration and data analysis, Document XMM-SOC-CALTN-0018-2-3, XMM Science Operation Centre, Villafranca del Castillo, 2004, available at http://xmm.vilspa.esa.es/docs/ documents/CAL-TN-0018-2-3.pdf

Krautter, J., Zickgraf, F.-J., Appenzeller, I., et al. 1999, A\&A, 350, 743

Lazzati, D., \& Perna, R. 2002, MNRAS, 330, 383

Le Fèvre, O., Vettolani, G., Paltani, et al. 2004, A\&A, 428, 1043

Malesani, D., D’Avanzo, P., Melandri, A., et al. 2004, GCN, 2685

Malesani, D., Tagliaferri, G., Chincarini, G., et al. 2004, ApJ, 609, L5

Mereghetti, S., Götz, D., Tiengo, A., et al. 2003, ApJ, 590, L73

Mereghetti, S., Götz, D., Beckmann, V., et al. 2003, A\&A, 411, L291

Mereghetti, S., Götz, D., Beck, M., et al. 2004a, GCN, 2670

Mereghetti, S., Götz, D., Beck, M., et al. 2004b, GCN, 2672

Moran, L., Mereghetti, S., Götz, D., et al. 2005, A\&A, in press [arXiv: astro-ph/0411565]

Noll, S., Mehlert, D., Appenzeller, I., et al. 2004, A\&A, 418, 885

Owens, A., Guainazzi, M., Oosterbroek, T., et al. 1998, A\&A, 339, L37

Piran, T. 2004, Rev. Modern Phys., accepted [arXiv: astro-ph/0405503]

Piro, L. 2004, in Gamma-Ray Bursts in the Afterglow Era (ASP), 312, in press [arXiv: astro-ph/0402638]

Predehl, P., \& Schmitt, J. H. M. M. 1995, A\&A, 293, 889

Price, P. A., Berger, E., Reichart, D. E., et al. 2002, ApJ, 572, L51

Prochaska, J. X., Bloom, J. S., Chen, H.-W., et al. 2004, ApJ, 611, 200

Read, A. 2004, Document XMM-SOC-CAL-SRN-167, available from http://xmm.vilspa.esa.es/docs/documents/

Reeves, J. N., Osborne, J. P., Watson, D., et al. 2002, Nature, 416, 512 Rodriguez-Pascual, P., \& Gonzalez-Riestra, R. 2004, GCN, 2688

Rodriguez-Pascual, P., \& Juarez, B. 2004, GCN, 2678

Sari, R., Piran, T., \& Narayan, R. 1998, ApJ, 497, L17

Sari, R., Piran, T., \& Halpern, J. 1999, ApJ, 519, L17

Schartel, N. 2004, GCN, 2700

Schlegel, E. M., Finkbeiner, D. P., \& Davis, M. 1998, ApJ, 500, 525

Simoncelli, A., D’ Avanzo, P., Campana, S., et al. 2004, GCN, 2549

Smith, D. A., Levine, A. M., Remillard, R., Hurley, K., \& Cline, T. 2000, GCN, 861

Soderberg, A, \& Frail, D. 2004, GCN, 2689

Stetson, P. B. 1987, PASP, 99, 191

Stratta, G., Fiore, F., Antonelli, L. A., Piro, L., \& De Pasquale, M. 2004, ApJ, 608, 846

Strüder, L., Briel, U., Dennerl, K., et al. 2001, A\&A, 365, L18

Tanvir, N., Priddey, R., Hughes, M., et al. 2004a, GCN, 2682

Tanvir, N., Priddey, R., Levan, A., et al. 2004b, GCN, 2684

Tiengo, A., Mereghetti, S., Ghisellini, G., et al. 2003, A\&A, 409, 983

Tiengo, A., Mereghetti, S., \& De Luca, A. 2004, GCN, 2548

Turner, M. J. L., Abbey, A., Arnaud, M., et al. 2001, A\&A, 365, L27

van Paradijs, J., Kouveliotou, C., \& Wijers, R. A. M. J. 2000, ARA\&A, 38, 379

Watson, D., Reeves, J. N., Osborne, J. P., et al. 2002a, A\&A, 395, L41

Watson, D., Reeves, J. N., Osborne, J. P., et al. 2002b, A\&A, 393, L1

Watson, D., Reeves, J. N., Hjorth, J., et al. 2003, ApJ, 595, L29

Watson, D., Hjorth, J., Levan, A., et al. 2004, ApJ, 605, L101 